\title{
The parameters for quantitative analysis of mutation rates with cultured mammalian somatic cells*
}

\author{
Jiliang Fu**, I.-Chian $\mathrm{Li}$ and E.H.Y. Chu \\ Department of Human Genetics, University of Michigan Medical School, Ann Arbor, MI 48109 \\ (U.S.A.)
}

(Accepted 3 July 1982)

In their classic paper which demonstrated the preadaptive origin of phage resistance in bacteria, Luria and Delbrück (1943) proposed the use of the fluctuation test to estimate mutation rates in a bacterial population. Subsequently, a number of investigators have examined this problem from various angles, including formal derivations (Newcombe, 1948), mathematical models (Stocker, 1949), mutant distribution (Lea and Coulson, 1950) and statistical theories (Armitage, 1952, 1953). The study in this problem has been continued until recently (Kondo, 1972; Gilbert, 1980), all dealing with the formulae and methods for estimating mutation rates in bacteria. Since the initial demonstration of experimental mutagenesis in cultured mammalian cells (Chu and Malling, 1968; Kao and Puck, 1968), fluctuation analysis has been applied for quantitative estimation of mutation rates (Shapiro et al., 1972; Morrow, 1975). There has nevertheless been a great disparity among published rate estimates from experiments with a variety of cells and genetic marker systems; using the same genetic marker in the same cell types, differences in mutation rates have even been found in different experiments by the same laboratory. For instance, Albertini and DeMars (1973) estimated the spontaneous mutation rate to 8 -azaguanine resistance in human diploid fibroblasts and obtained values in 5 Expts. varying by a factor of $14\left(0.788 \times 10^{-6}\right.$ to $1.115 \times 10^{-5}$ per cell per generation.

There are at least 3 sources of error that may be responsible for such differences. The most obvious would be the sampling error, but it is too big a range of values to be the sole source. The second source of error may be biological, which has been described in some detail by Abbondandolo (1977). The third type of error may originate from differences in experimental design. There are at least 5 different methods for the estimation of mutation rates, namely, the mean, the upper quartile, the median, the $P_{\mathrm{o}}$ and the maximum likelihood methods (Luria and Delbrück, 1943; Newcombe, 1948; Lea and Coulson, 1950; Armitage, 1952; 1953; Kondo,

*Research supported by NIH Grant CA 26803.

${ }^{* *}$ On leave from the School of Public Health, Sichuan Medical College, Chengdu, Sichuan (China). 
1972). The order of statistical preference in choosing a method has been evaluated by us ( $\mathrm{Li}$ et al., 1982b). We limit the present communication to a discussion of the various parameters involved in rate estimates of mutations in monolayer cultures of mammalian cells by the $P_{0}$ method. The effect of phenotypic delay on the rate estimation is not considered here because the problem has been dealt with statistically or experimentally by others (Newcombe, 1948; Armitage, 1952, 1953; O'Neill et al., 1981). As a consequence of this exercise, some practical procedures are recommended which may lead to a more reliable estimation of mutation rates.

\section{The parameters}

Luria and Delbrück's basic assumption was that gene mutations occur very rarely and follow a Poisson distribution in parallel bacteria cultures. The formula they used for calculating the mutation rate from the fluctuation test is:

$$
P_{\mathrm{o}}=\mathrm{e}^{-\mu} N
$$

Where $\mu$ is the number of mutations per time unit i.e., the mutation rate, $P_{\mathrm{o}}$ is the proportion of parallel cultures without any mutant colonies, $N$ is the population size and $e$ is the base of natural logarithms. Mammalian cells grow slower than bacteria. In addition, there are many factors that may affect the recovery of mutants from a cell population. In the following discussion, we shall consider, in sequence, several parameters involved in fluctuation analysis with cultured mammalian cells.

$P_{\mathrm{o}}$, the proportion of parallel cultures without any mutant; $C$, the number of parallel cultures in a fluctuation test; $N_{\mathrm{o}}$, the initial population size of a culture; $N$, the final population size per parallel culture when the selective agent is added; $D$, the total number of dishes used in the experiment; $K$, the number of parallel cultures containing mutants in a total of $C$ cultures; $n$, the maximum cell number that can be inoculated to 1 dish, without producing any cell density effect on mutant recovery.

\section{The fraction of parallel cultures without mutant(s) - the value of $\boldsymbol{P}_{\mathrm{o}}$}

Before starting a fluctuation test, a rough estimate of the mutation rate for the genetic marker under study may be obtained from either the literature or preliminary tests. Sometimes the average of mutation rates known for several other markers in the same cell line can be taken as a predicted value of $\mu$.

From eqn. [1], when $\mu N=1$, i.e., the average chance to have a mutation per culture equals one, $P_{\mathrm{o}}$ is 0.37 . This might be a suitable point for the estimation of $\mu$. $\mu$ can be regarded as a constant for a given marker. Since $P_{\mathrm{o}}$ is a function of $N$, $P_{\mathrm{o}}$ can be controlled by adjusting $N$. In order to obtain a better estimation of $\mu, P_{\mathrm{o}}$ should not be approaching 0 or 1 . 


\section{The choice of $C$, the number of parallel cultures}

The greater the number of parallel cultures one uses, the more reliable is the estimation of mutation rates. Because of the cost and technical difficulties, a minimum $C$ must be chosen without sacrificing the reliability of rate estimates.

Suppose $K$ is the number of cultures which contain mutants in a total number of $C$ parallel cultures, then:

$$
P_{\mathrm{o}}=\frac{C-K}{C} \text { or } \quad C=\frac{K}{1-P_{\mathrm{o}}}
$$

Here $K$ is a positive integer, less than $C$ but larger than zero. The value of $K$ may vary from $K$ to $K \pm 1, K \pm 2, K \pm 3, \ldots$ If it is stipulated that the relative sampling error of $K$ must not be larger than $10 \%$, then:

$$
\left|\frac{(K \pm 1)-K}{K}\right|=\frac{1}{K} \leq 0.1 \text { or } K \geq 10
$$

Put the value $K \geq 10$ into eqn. [2], one obtains:

$$
C \geq \frac{10}{1-P_{\mathrm{o}}}
$$

The C's so derived will give estimated mutation rates to vary around 4-fold as judged from the top limits to bottom limits in terms of confidence intervals of mutation rates. This relationship has been elaborated elsewhere ( $\mathrm{Li}$ et al., 1982a). For example, when $P_{\mathrm{o}}=0.37, C$ can be calculated as follows:

$$
\begin{aligned}
& C \geq \frac{10}{1-0.37} \\
& C \geq 16
\end{aligned}
$$

In other words, if $\mu N=1, P_{\mathrm{o}}=0.37$, the minimum $C$ should be 16 for a reliable estimation within a range of 4 -fold variation of $\mu$. Fig. 1 shows the relationship between $N$ and $\mu$ for different values of $P_{\mathrm{o}}$ when $C$ is at the minimum. This relationship is based on eqns. [1] and [2]. Once a value of $\mu$ is predicted, a vertical line can be drawn that will come across the oblique line of an expected $P_{\mathrm{o}}$ at a definitive point. A horizontal line can then be extended from the interception point to the ordinate to find a corresponding value of $N$.

For example, if a spontaneous mutation rate for a given genetic marker is predicted to be $5 \times 10^{-8}$ per cell generation and a $P_{\mathrm{o}}$ is expected to be 0.80 , a corresponding $N$ of $4.5 \times 10^{6}$ can be found. It means that the final population size of $4.5 \times 10^{6}$ cells per culture and 50 or more parallel cultures must be used for a reliable mutation rate estimate. Alternatively, the final population may be reduced to $2.2 \times 10^{4}$ to $4.5 \times 10^{4}$ cells per parallel culture when the mutation rate is elevated to within a range of $5 \times 10^{-6}$ to $1 \times 10^{-5}$ per cell per generation, such as in the cases of induced mutations or hypermutable cell strains. 


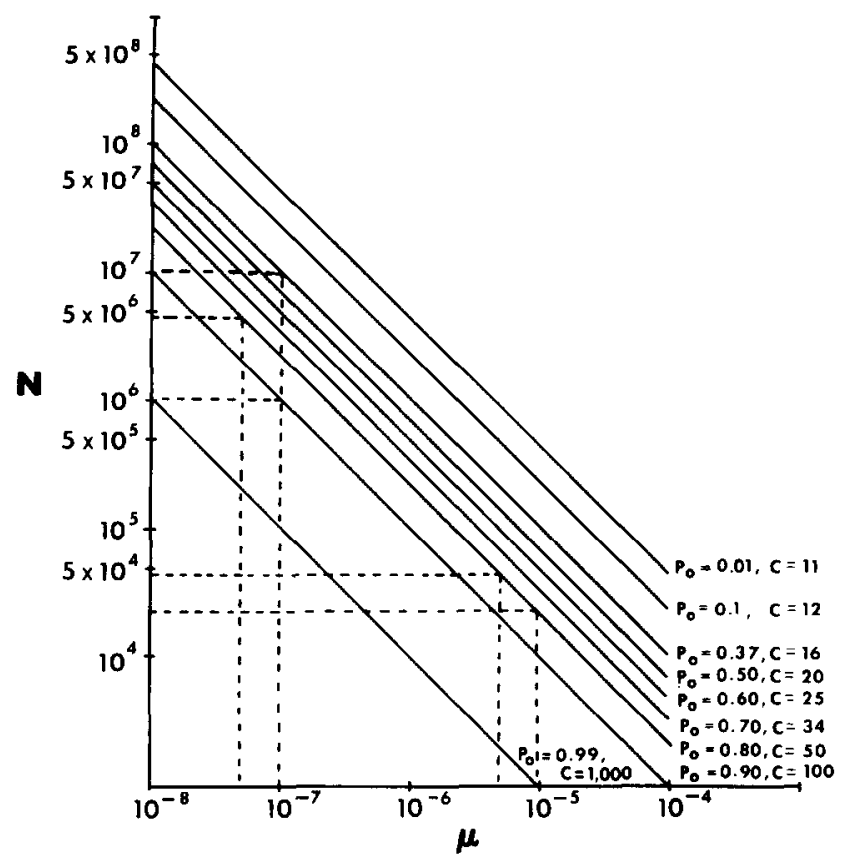

Fig. 1. The relationship between $N$ and $\mu$ for different values of $P_{\mathrm{o}}$ at minimum $C$.

The total number of dishes, $D$

In mutagenic experiments with cultured mammalian cells, $D$, the total number of dishes used, is limited by manpower and material resources, including media, serum, petri dishes, incubator space, etc. Therefore $D$ can be taken as the main reference in determining the feasibility of an experiment.

In a fluctuation test with bacteria, $D$ usually equals $C$, meaning that all of the bacteria can be inoculated in one dish as a parallel culture. In tests with mammalian cells, however, the number of cells inoculated in each dish is limited by inter- or intra-colony growth inhibition as well as by the cell density effect on mutant recovery.

From reconstruction experiments for determining the cell-density effect on mutant recovery, the maximum cell number than can be inoculated into each dish should be $n$. The value of $D$ follows the formula:

$$
D=C \cdot \frac{N}{n}
$$

For example, when $N=10^{7}$ cells per parallel culture, $C=20, n=10^{5}$ cells per dish, $D$ can be calculated as follows:

$$
D=20 \times \frac{10^{7}}{10^{5}}=2000
$$


From eqn. [3], it can also be seen that the most effective way to reduce the value of $D$ is to increase the value of $n$. This can be achieved by choosing a marker, such as ouabain resistance, which has a negligible cell-density effect on mutant recovery. In other situations, this can also be achieved by adding to the culture medium some appropriate inhibitor of metabolic cooperation between wild-type cells and mutants (Diamond et al., 1979; Yotti et al., 1979). On the other hand, $D$ can be reduced by regulating $C$ and $N$. For example, if a predicted mutation rate for a given marker is $10^{-7}$ and $\mathbf{n}$ is $5 \times 10^{5}$ cells per dish, 2 alternative $N$ 's, e.g. $N_{1}=4 \times 10^{6}$ and $N_{2}=10^{6}$ could be chosen while keeping $K=10$. From eqns. [1] and [2]:

$$
\begin{aligned}
& C_{1}=\frac{10}{1-\mathrm{e}^{-\left[10^{\left.-\cdot \cdot 4 \cdot 10^{*}\right]}\right.}}=\frac{10}{1-\mathrm{e}^{-0.4}}=30 \\
& C_{2}=\frac{10}{1-\mathrm{e}^{-\left[10^{-\cdot \cdot} \cdot 10^{\circ}\right]}}=\frac{10}{1-\mathrm{e}^{-0.1}}=100
\end{aligned}
$$

Substituting $C_{1}$ and $C_{2}$ into eqn. [3]:

$$
\begin{aligned}
& D_{1}=C_{1} \cdot \frac{N_{1}}{n}=30 \times \frac{4 \times 10^{6}}{5 \times 10^{5}}=30 \times 8=240 \\
& D_{2}=C_{2} \cdot \frac{N_{2}}{n}=100 \times \frac{10^{6}}{5 \times 10^{5}}=100 \times 2=200
\end{aligned}
$$

Hence if $C_{0}$ is adopted, $20 \%$ of dishes may be saved.

From Fig. 1, depending on a predicted $\mu$ and an expected $P_{\mathrm{o}}$, different combinations of $N$ and $C$ can be obtained. An appropriate $D$ can then be calculated from eqn. [3]. This procedure determines whether a fluctuation test leading to a reliable estimation of mutation rate is feasible or not.

The size of initial inoculum per culture, $N_{\mathrm{o}}$

In order to reduce the chance of including a preexisting mutant cell in the inoculum, it is thought that $N_{\mathrm{o}}$ should be as small as possible, preferably one cell from which to expand to a large population. This is easily accomplished for bacteria, but is not so easily done for a mammalian cell population. The time required for one bacterium to grow up to $10^{8}$ cells is only about $9 \mathrm{~h}$, but is as long as 13-26 days for the mammalian cell, even though in both instances the total number of generations elapsed is 26.6. In addition, physical space within a culture vessel and nutritional requirements by the mammalian cells further limit the maximum population size per culture. In practice, cell populations have to be repeatedly dispersed to maintain an exponential growth, an important condition to ensure an equal opportunity for every cell to mutate and multiply. What makes things worse is that the probability of contamination of parallel cultures will increase upon subculturing. It is desirable then to shorten the growth period and reduce the need for subculture 
by increasing the value of $N_{\mathrm{o}}$ while taking a somewhat increased risk. For example, if $10^{4}$ cells are inoculated into each parallel culture and if it is known that the original cell population has a mutant frequency of $10^{-7}$, the risk for introducing a mutant into a culture is only one in one thousand, which is usually acceptable. However, this may be considered too high a risk in some other instances. If this is the case, the following procedure may be carried out.

We may give $g$ as the number of generations needed for an original population of $N_{\mathrm{o}}$ to grow to a population size of $N$, i.e.:

$$
\begin{gathered}
N=2^{g} \cdot N_{\circ} \\
2^{g}=\frac{N}{N_{\circ}} \\
g=\frac{\ln \left(N / N_{\circ}\right)}{\ln 2}
\end{gathered}
$$

If one mutant cell was inoculated into a parallel culture, as the population increases to $N$ the total number of mutant cells in the culture will be $2^{g}$. Thus, any parallel culture containing $2^{g}$ or more than $2^{g}$ mutant cells should be considered as having a preexisting mutant and should be discarded. In the case of a culture increasing its size from $10^{4}$ to $10^{7}$ cells, the number of generations it passes through is:

$$
g=\frac{\ln \left(N / N_{0}\right)}{\ln 2}=\frac{\ln \left(10^{7} / 10^{4}\right)}{\ln 2}=9.97 \simeq 10
$$

It means that the total mutants from the preexisting mutant cell should be about $2^{10}$, i.e. about 1000. In the case where one particular parallel culture has 1000 or more mutant colonies, we may simply disregard it before analyzing the result from the rest of the experiment.

\section{Summary and conclusions}

The sequence of the decision-making process for determining the various parameters in designing a fluctuation experiment with cultured mammalian cells may be summarized in Fig. 2. First, a value of $\mu$ is predicted from the literature or a preliminary experiment. Second, the values of $N$ and $N_{\circ}$ are determined on the basis of the experimental conditions and the size of the expected $P_{o}$. Third, the minimum $C$ needed for a reliable test can be calculated from eqn. [2]. If necessary, $C$ can be increased slightly to compensate for any loss of cultures due to contamination or other unexpected errors, and to serve as an extra supply if the actual mutation rate is lower than predicted. Finally, $D$ can be calculated from eqn. [3] after knowing the size of $n$ by a reconstruction experiment. We believe that this is the first 


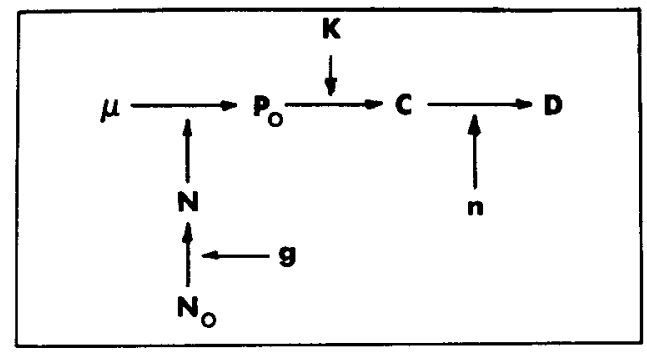

Fig. 2. The relationship between all the parameters related to a fluctuation test with mammalian somatic cells in culture.

time that the parameters in a fluctuation experiment with mammalian cells have been discussed in detail. The relationships among all of the parameters thus revealed have provided us a practical guide for designing experiments on quantitative mutagenesis with cultured mammalian cells. A reliable estimation of somatic mutation rates is obviously important for assessing the genetic load and the genetic risks to man by environmental agents.

\section{References}

Abbondandolo, A. (1977) Prospects for evaluating genetic damage in mammalian cells in culture, Mutation Res., 42, 279-298.

Albertini, R.J., and R. DeMars (1973) Somatic cell mutation, detection and quantification of X-rayinduced mutation in cultured, diploid human fibroblasts, Mutation Res., 18, 199-224.

Armitage, P.J. (1952) The statistical theory of bacterial populations subject to mutation, J. Roy. Statist . Soc., B, 14, 1-40.

Armitage, P.J. (1953) Statistical concepts in the theory of bacteria mutation, J. Hyg., 51, $162-184$.

Chu, E.H.Y., and H.V. Malling (1968) Mammalian cell genetics, II. Chemical induction of specific locus mutations in Chinese hamster cells in vitro, Proc. Natl. Acad. Sci. (U.S.A.), 61, 1306-1312.

Diamond, L., T.G. O'Brien and G. Rovera (1978) Tumor promoters: Effects on proliferation and differentiation of cells in culture, Life Sci., 23, 1979--1988.

Gilbert, R.E. (1980) The analysis of fluctuation tests, Mutation Res., 74, 283-289.

Kao, F.-T., and T. Puck (1968) Genetics of somatic mammalian cells, VII. Induction and isolation of nutritional mutants in Chinese hamster cells, Proc. Natl. Acad. Sci (U.S.A.), 60, 1275-1281.

Kondo, S. (1972) A theoretical study on spontaneous mutation rate, Mutation. Res., 14, 365-374.

Lea, D.E., and C.A. Coulson (1950) The distribution of the numbers of mutants in bacteria populations, J. Genet., 49, 264-285.

Li, I.-Chian, J. Fu, Y.-T. Hung and E.H.Y. Chu (1982a) The reliability of mutation rates estimated by fluctuation analysis in cultured mammalian cells: Effect of the number of parallel cultures and the final population size on the variance of the rate estimates, Genetics, 100, s40-41.

Li, I.-C., J. Fu, Y.-T. Hung and E.H.Y. Chu (1982b) Evaluation of methods for the estimation of mutation rates in cultured mammalian cell populations, Abstract, 33rd Annual Meeting of the American Society of Human Genetics, Am. J. Hum. Genet., in press. 
Luria, S.E., and M. Delbrück (1943) Mutations of bacteria from virus sensitivity to virus resistance, Genetics, 28, 491-511.

Morrow, J. (1975) On the relationship between spontaneous mutation rates in vivo and in vitro, Mutation Res., 33, 367-372.

Newcombe, H.B. (1948) Delayed phenotypic expression of spontaneous mutation in Escherichia coli, Genetics, 33, 447-476.

O'Neill, J.P., P.A. Brimer and A.W. Hsie (1981) Fluctuation analyses of spontaneous mutation to 6-thioguanine resistance in Chinese hamster ovary cells in culture, Mutation Res., 82, 343-353.

Shapiro, N.I., A.E. Khalizev, E.V. Luss, M.I. Marshak, O.N. Petrova and N.B. Varshaver (1972) Mutagenesis in cultured mammalian cells, I. Spontaneous gene mutations in human and Chinese hamster cells, Mutation Res., 15, 203-214.

Stocker, B.A.D. (1949) Measurements of rate of mutation of flagellar antigenic phase in Salmonella typhimurium, J. Hyg., 47, 398-413.

Yotti, L.P., C.C. Chang and J.E. Trosko (1979) Elimination of metabolic cooperation in Chinese hamster cells by a tumor promoter, Science, 206, 1089-1091. 